\title{
NEW DIFFERENTIAL SUBORDINATIONS OBTAINED BY USING A DIFFERENTIAL-INTEGRAL RUSCHEWEYH-LIBERA OPERATOR
}

\author{
GEORGIA IRINA OROS
}

Received 21 October, 2019

\begin{abstract}
In this paper, the operator denoted by $D^{m}: A \rightarrow A$, is defined by $D^{m}[f](z)=(1-$ $\lambda) R^{m}[f](z)+\lambda L^{m}[f](z), z \in U$, a differential-integral operator, where $R^{m}$ is Ruscheweyh differential operator and $L^{m}$ is Libera integral operator. By using the operator $D^{m}$ the class of univalent functions denoted by $M(m, \lambda, \alpha), 0 \leq \lambda \leq 1,0 \leq \alpha<1$, is defined and several differential subordinations are studied.
\end{abstract}

2010 Mathematics Subject Classification: 30C20; 30C45; 30A40

Keywords: analytic function, differential operator, integral operator, convex function, univalent function, best dominant, differential subordination

\section{INTRODUCTION AND PRELIMINARIES}

The concept of differential subordination was introduced in [5,6] by S.S. Miller and P.T. Mocanu. This theory had a great impact in the field of complex analysis and developed rapidly. Some recent published work can be seen in [7, 9, 10, 10-12].

Studying subordination properties associated with various operators is a topic of interest at this time. Many researchers continue the study of the operators and classes of univalent functions using the methods of the theory of differential subordination. In [17], the authors have generalized and improved some of the previously published results by introducing a general family of analytic and bi-univalent functions in the open unit disk defined by applying the principle of differential subordination between analytic functions and the Tremblay fractional derivative operator. By making use of the linear operator introduced and studied earlier by Srivastava and Attiya, in paper [16] the authors have investigated classes of admissible functions and have presented properties of the third-order differential subordinations establishing various sandwich-type theorems for a class of univalent analytic functions involving the celebrated Srivastava-Attiya transform. In another recent paper [15], the authors introduce and investigate the Fekete-Szegô functional associated with a new subclass of analytic functions defined by using the principle of quasi-subordination between analytic functions, some sufficient conditions for functions belonging to this class 
being also derived. The Fekete-Szegô coefficient functional for functions in a newly introduced class of normalized analytic functions in the open unit disc were obtained in paper [18]. Various integral and convolution characterizations, coefficient estimates and differential subordination results for functions belonging to that class were also investigated in the paper. By making use of the principle of differential subordination and the Dziok - Srivastava convolution operator, in paper [19] the authors introduce and investigate three interesting subclasses of analytic and univalent functions in the open unit disk. Inclusion relationships for those classes and that these classes are closed under convolution with convex functions is also proved.

In this paper a new class of univalent functions and a new operator are introduced. The new operator is used for obtaining subordination results related to this class. Similar work containing subordination results related to a class of univalent functions obtained by the use of an operator introduced by using a differential operator and an integral one has been published recently [12] and inspired the results shown in this paper. Also, following the same idea, in paper [13], the author introduces new classes obtained by using the linear operator defined with the Sălăgean differential operator and the generalized Alexander operator, studying the characteristics and other properties of these classes and obtaining Fekete-Szegó functional for these classes.

We use the following well known notations and results:

Denote by $U$ the unit disc of the complex plane

$$
U=\{z \in \mathbb{C}:|z|<1\} .
$$

Let $\mathcal{H}(U)$ be the space of holomorphic functions in $U$ and let

$$
A_{n}=\left\{f \in \mathcal{H}(U): f(z)=z+a_{n+1} z^{n+1}+a_{n+2} z^{n+2}+\ldots, z \in U\right\},
$$

with $A_{1}=A$.

Let $S=\{f \in A: f$ is univalent in $U\}$ be the class of holomorphic and univalent functions in the open unit disc $U$ with the conditions $f(0)=0$ and $f^{\prime}(0)=1$, that is the holomorphic and univalent functions with the following power series development

$$
f(z)=z+a_{2} z^{2}+\ldots, z \in U .
$$

For $a \in \mathbb{C}$ and $n \in \mathbb{N}^{*}$ we denote by

$$
\mathcal{H}[a, n]=\left\{f \in \mathcal{H}(U): f(z)=a+a_{n} z^{n}+a_{n+1} z^{n+1}+\ldots, z \in U\right\} .
$$

Denote by

$K=\left\{f \in A: \operatorname{Re}\left(\frac{z f^{\prime \prime}(z)}{z f^{\prime}(z)}+1\right)>0, z \in U\right\}$ the class of normalized convex functions in $U$ and let

$$
S^{*}=\left\{f \in A: \operatorname{Re} \frac{z f^{\prime}(z)}{f(z)}>0, z \in U\right\} \text { denote the class of starlike functions in } U .
$$




\section{Definition of subordination ([7, p.4])}

If $f$ and $g$ are analytic functions in $U$, then we say that $f$ is subordinate to $g$, written $f \prec g$ or $f(z) \prec g(z)$, if there is a function $w$, analytic in $U$, with $w(0)=0$ and $|w(z)|<1$ for all $z \in U$ such that $f(z)=g(w(z))$ for $z \in U$. If $g$ is univalent, then $f \prec g$ or $f(z) \prec g(z)$ if and only if $f(0)=g(0)$ and $f(U) \subset g(U)$.

\section{Definition of second-order differential subordination ([7, p.16])}

Let $\psi: \mathbb{C}^{3} \times U \rightarrow \mathbb{C}$ and let $h$ be univalent in $U$. If $p$ is analytic in $U$ and satisfies the second-order differential subordination

(i) $\psi\left(p(z), z p^{\prime 2} p^{\prime \prime}(z) ; z\right) \prec h(z), z \in U$ then $p$ is called a solution of the differential subordination.

The univalent function $q$ is called a dominant of the solutions of the differential subordination, or more simply, a dominant if $p \prec q$ for all $p$ satisfying (i).

A dominant $\widetilde{q}$ that satisfies $\widetilde{q} \prec q$ for all dominants $q$ of (i) is said to be the best dominant of (i). (Note that the best dominant is unique up to a rotation of $U$ ).

If we require the more restrictive condition $q \in \mathcal{H}[a, n]$ then $p$ is called an $(a, n)$ solution, $q$ an $(a, n)$-dominant and $\widetilde{q}$ the best $(a, n)$-dominant.

To prove our main results, we need the following definitions and lemmas:

Definition 1 (Ruscheweyh operator [14]). For $f \in A, m \in \mathbb{N}$, the operator $R^{m}$ is defined by $R^{m}: A \rightarrow A$,

$$
\begin{aligned}
& R^{0}[f](z)=f(z) \\
& R^{1}[f](z)=z \cdot\left[R^{0} f(z)\right]^{\prime}=z f^{\prime}(z) \\
& \vdots \\
& (m+1) R^{m+1}[f](z)=z \cdot\left[R^{m} f(z)\right]^{\prime}+m R^{m} f(z), z \in U .
\end{aligned}
$$

Remark 1. If $f \in A, f(z)=z+a_{2} z^{2}+a_{3} z^{3}+\ldots=z+\sum_{k=2}^{\infty} a_{k} z^{k}$, then

$$
R^{m}[f](z)=z+\sum_{k=2}^{\infty} a_{k} C_{m+k-1}^{m} z^{k}=z+\sum_{k=2}^{\infty}\left[\begin{array}{c}
m+k-1 \\
m
\end{array}\right] a_{k} z^{k} .
$$

Definition 2 ([1, Definition 1]). For $f \in A, m \in \mathbb{N}, L^{m}: A \rightarrow A$ is defined by

$$
\begin{aligned}
& L^{0}[f](z)=f(z) \\
& L^{1}[f](z)=\frac{2}{z} \int_{0}^{z} L^{0}[f](t) d t=\frac{2}{z} \int_{0}^{z} f(t) d t \\
& \vdots \\
& L^{m}[f](z)=\frac{2}{z} \int_{0}^{z} L^{m-1}[f](t) d t, z \in U .
\end{aligned}
$$


Remark 2. a) For $m=1$,

$$
L^{1}[f](z)=\frac{2}{z} \int_{0}^{z} f(t) d t
$$

which is Libera integral operator [5].

b) If $f \in A, f(z)=z+a_{2} z_{2}+a_{3} z^{3}+\ldots=z+\sum_{k=2}^{\infty} a_{k} z^{k}$, then

$$
\begin{aligned}
L^{m}[f](z) & =\frac{2}{z} \int_{0}^{z} L^{m-1}[f](t) d t \\
& =\frac{2}{z} \int_{0}^{z}\left[t+\sum_{k=2}^{\infty} \frac{2^{m-1}}{(k+1)^{m-1}} \cdot a_{k} t^{k}\right] d t \\
& =\frac{2}{z}\left[\frac{z^{2}}{2}+\sum_{k=2}^{\infty} \frac{2^{m-1}}{(k+1)^{m-1}} \cdot a_{k} \cdot \frac{z^{k+1}}{k+1}\right] \\
& =z+\sum_{k=2}^{\infty} \frac{2^{m}}{(k+1)^{m}} \cdot a_{k} z^{k} .
\end{aligned}
$$

c) For $f \in A, m \in \mathbb{N}$, we obtain

$$
z\left(L^{m}[f](z)\right)^{\prime}=2 L^{m-1}[f](z)-L^{m}[f](z), z \in U .
$$

Lemma A. (Hallenbeck and Ruscheweyh [7, Th. 3.1.b, p. 71], [2]) Let h be convex in $U$, with $h(0)=a, \gamma \neq 0$ and $\operatorname{Re} \gamma \geq 0$. If $p \in \mathcal{H}[a, n]$ and

$$
p(z)+\frac{1}{\gamma} z p^{\prime}(z) \prec h(z), z \in U,
$$

then

$$
p(z) \prec q(z) \prec h(z), z \in U,
$$

where

$$
q(z)=\frac{\frac{\gamma}{n}}{z^{\frac{\gamma}{n}}} \int_{0}^{z} h(t) t^{\frac{\gamma}{n}-1} d t, z \in U .
$$

The function $q$ is convex and is the best $\left(a_{n}\right)$-dominant.

Lemma B. ([7, Th. 3.4.h, p. 132]) Let $q$ be univalent in $U$ and let $\theta$ and $\phi$ be analytic in a domain D containing $q(U)$, with $\phi(w) \neq 0$ when $w \in q(U)$. Set

$$
Q(z)=z q^{\prime}(z) \phi[q(z)], \quad h(z)=\theta[q(z)]+Q(z)
$$

and suppose that either

(i) $h$ is convex, or

(ii) $Q$ is starlike.

In addition, assume that 
(iii) $\operatorname{Re} \frac{z h^{\prime}(z)}{Q(z)}>\operatorname{Re}\left[\frac{\theta^{\prime}[q(z)]}{\phi[q(z)]}+\frac{z Q^{\prime}(z)}{Q(z)}\right]>0$.

If $p$ is analytic in $U$, with $p(0)=q(0), p(U) \subset D$ and

$$
\theta[p(z)]+z p^{\prime}(z) \cdot \phi[p(z)] \prec \theta[q(z)]+z q^{\prime}(z) \cdot \phi[q(z)]=h(z),
$$

then $p(z) \prec q(z)$ and $q$ is the best dominant.

\section{MAIN RESULTS}

In this paper, we define a differential-integral operator, $D^{m}: A \rightarrow A$, we introduce a class of holomorphic univalent functions and we study several differential subordinations.

Definition 3. Let $m \in \mathbb{N}, 0 \leq \lambda \leq 1$, denote by $D^{m}: A \rightarrow A$,

$$
D^{m}[f](z)=(1-\lambda) R^{m}[f](z)+\lambda L^{m}[f](z),
$$

where $R^{m}$ is given by (1) and $L^{m}$ is given by (3).

Remark 3. a) If $f \in A, f(z)=z+\sum_{k=2}^{\infty} a_{k} z^{k}$ and using (2) and (4), we have

$$
\begin{gathered}
D^{m}[f](z) \\
=(1-\lambda)\left(z+\sum_{k=2}^{\infty}\left[\begin{array}{c}
m+k-1 \\
m
\end{array}\right] a_{k} z^{k}\right)+\lambda\left(z+\sum_{k=2}^{\infty} \frac{2^{m}}{(k+1)^{m}} a_{k} z^{k}\right) \\
=z+\sum_{k=2}^{\infty} a_{k} z^{k}\left\{\left[\begin{array}{c}
m+k-1 \\
m
\end{array}\right](1-\lambda)+\lambda \frac{2^{m}}{(k+1)^{m}}\right\} .
\end{gathered}
$$

b) For $\lambda=1$, differential-integral operator $D^{m}$ coincides with Libera integral operator (Definition 2).

c) For $\lambda=0$, differential-integral operator $D^{m}$ coincides with differential operator Ruscheweyh (Definition 1).

Definition 4. If $0 \leq \alpha<1,0 \leq \lambda \leq 1, m \in \mathbb{N}$, we let $M(m, \lambda, \alpha)$ stand for the class of functions $f \in A$, which satisfy the inequality

$$
\operatorname{Re}\left(D^{m}[f](z)\right)^{\prime}>\alpha, z \in U,
$$

where the differential-integral operator $D^{m}[f]$ is given by (6).

Remark 4. a) For $m=0,0 \leq \lambda \leq 1, \alpha=0$, the operator $D^{m}[f]$ becomes

$$
D^{0}[f](z)=(1-\lambda) R^{0}[f](z)+\lambda L^{0}[f](z)=(1-\lambda) f(z)+\lambda f(z)=f(z), z \in U,
$$

then $M(m, \lambda, \alpha)$ becomes

$$
M(0, \lambda, 0)=R=\left\{f \in A: \operatorname{Re} f^{\prime}(z)>0, z \in U\right\}
$$

called the class of functions with bounded rotation. 
This class of functions was studied by J.W. Alexander [1] and he proved that $R \subset S$, J. Kurzyz [3] and P.T. Mocanu [8] have proved that $R \not \subset S^{*}$. A more systematic study of the class $R$ was done by Mac Gregor [4].

b) For $m=0,0 \leq \alpha<1,0 \leq \lambda \leq 1$, we have

$$
M(0, \lambda, \alpha)=\left\{f \in A: \operatorname{Re} f^{\prime}(z)>\alpha\right\} \subset R .
$$

Theorem 1. The set $M(m, \lambda, \alpha)$ is convex.

Proof. Let the functions

$$
f_{j}(z)=z+\sum_{k=2}^{\infty} C_{k j} z^{j}, j=1,2, z \in U
$$

where

$$
C_{k j}=a_{k j}\left\{(1-\lambda)\left[\begin{array}{c}
m+k-1 \\
m
\end{array}\right]+\frac{2^{m}}{(k+1)^{m}}\right\},
$$

be in the class $M(m, \lambda, \alpha)$. It is sufficient to show that the function

$$
h(z)=\mu_{1} f_{1}(z)+\mu_{2} f_{2}(z), z \in U,
$$

with $\mu_{1}, \mu_{2} \geq 0$ and $\mu_{1}+\mu_{2}=1$ is in $M(m, \lambda, \alpha)$.

Since $h(z)=\mu_{1} f_{1}(z)+\mu_{2} f_{2}(z), z \in U$, then

$$
\begin{gathered}
D^{m}[h](z) \\
=z+\sum_{k=2}^{\infty}\left\{(1-\lambda)\left[\begin{array}{c}
m+k-1 \\
m
\end{array}\right]+\lambda \frac{2^{m}}{(k+1)^{m}}\right\}\left(\mu_{1} a_{k 1}+\mu_{2} a_{k 2}\right) z^{k} .
\end{gathered}
$$

Differentiating (9), we have

$$
\left(D^{m}[h](z)\right)^{\prime}=1+\sum_{k=2}^{\infty}\left\{(1-\lambda)\left[\begin{array}{c}
m+k-1 \\
m
\end{array}\right]+\lambda \frac{2^{m}}{(k+1)^{m}}\right\}\left(\mu_{1} a_{k-1}+\mu_{2} a_{k 2}\right) k z^{k-1} .
$$

Hence

$$
\begin{gathered}
\operatorname{Re}\left(D^{m}[h](z)\right)^{\prime} \\
=1+\mu_{1} \operatorname{Re} \sum_{k=2}^{\infty}\left\{(1-\lambda)\left[\begin{array}{c}
m+k-1 \\
m
\end{array}\right]+\lambda \frac{2^{m}}{(k+1)^{m}}\right\} a_{k 1} k z^{k-1} \\
+\mu_{2} \operatorname{Re}\left\{\sum_{k=2}^{\infty}(1-\lambda)\left[\begin{array}{c}
m+k-1 \\
m
\end{array}\right] \lambda \frac{2^{m}}{(k+1)^{m}}\right\} a_{k 2} k z^{k-1} .
\end{gathered}
$$

Since $f_{1}, f_{2} \in M(m, \lambda, \alpha)$, we have

$$
\begin{aligned}
& \mu_{1} \operatorname{Re} \sum_{k=2}^{\infty}\left\{(1-\lambda)\left[\begin{array}{c}
m+k-1 \\
m
\end{array}\right]+\lambda \frac{2^{m}}{(k+1)^{m}}\right\} a_{k 1} k z^{k-1}>\mu_{1}(\alpha-1) \\
& \mu_{2} \operatorname{Re} \sum_{k=2}^{\infty}\left\{(1-\lambda)\left[\begin{array}{c}
m+k-1 \\
m
\end{array}\right]+\lambda \frac{2^{m}}{(k+1)^{m}}\right\} a_{k 2} k z^{k-1}>\mu_{2}(\alpha-1)
\end{aligned}
$$


Using (11) and (12), we have

$$
\operatorname{Re}\left(D^{m}[h](z)\right)^{\prime}>1+\mu_{1}(\alpha-1)+\mu_{2}(\alpha-1)
$$

and since $\mu_{1}+\mu_{2}=1$, we deduce

$$
\operatorname{Re}\left(D^{m}[h](z)\right)^{\prime}>\alpha, z \in U
$$

i.e. $M(m, \lambda, \alpha)$ is convex.

Theorem 2. If $0 \leq \alpha<1,0 \leq \lambda \leq 1$ and $m \in \mathbb{N}$, then we have

$$
M(m, \lambda, \alpha) \subset M(m, \lambda, \delta),
$$

where $\delta=2 \alpha-1+2(1-\alpha) \ln 2, \delta \approx 0,62 \alpha+0,38<1$.

Proof. We will prove that $\delta \approx 0,62 \alpha+0,38<1$.

We know that $\ln 2 \approx 0,69$. From $0 \leq \alpha<1$, it follows

$$
\begin{gathered}
0 \leq 0,62 \alpha<0,62,0,38 \leq 0,62 \alpha+0,38<0,62+0,38, \\
0,38 \leq 0,62 \alpha+0,38<1,0,38 \leq \delta<1 .
\end{gathered}
$$

Consider the convex function

$$
h(z)=\frac{1+(2 \alpha-1) z}{1+z}, z \in U .
$$

For $z \in U, \operatorname{Re} h(z)>\alpha$ and $h(0)=1$.

We let $f \in M(m, \lambda, \alpha)$. From Definition 4, we have

$$
\operatorname{Re}\left(D^{m}[f](z)\right)^{\prime}>\alpha, z \in U .
$$

Let

$$
p(z)=\frac{D^{m}[f](z)}{z}, z \in U
$$

Using (7) in (15), we have

$$
p(z)=1+\sum_{k=2}^{\infty} a_{k} z^{k-1}\left\{\left[\begin{array}{c}
m+k-1 \\
m
\end{array}\right](1-\lambda)+\lambda \frac{2^{m}}{(k+1)^{m}}\right\}
$$

and $p(0)=1, p \in \mathcal{H}[1,1]$. From (15), we have

$$
D^{m}[f](z)=z p(z), z \in U
$$

Differentiating (16), we get

$$
\left(D^{m}[f](z)\right)^{\prime}=p(z)+z p^{\prime}(z), z \in U .
$$

Using (17), then (14) becomes

$$
\operatorname{Re}\left[p(z)+z p^{\prime}(z)\right]>\alpha, z \in U .
$$

Relation (18) can be written as a subordination of the form

$$
p(z)+z p^{\prime}(z) \prec h(z)=\frac{1+(2 \alpha-1) z}{1+z}, z \in U .
$$


Using Lemma $\mathrm{A}$, for $\gamma=1, n=1$, it follows

$$
p(z) \prec q(z),
$$

where

$$
q(z)=\frac{1}{z} \int_{0}^{z} \frac{1+(2 \alpha-1) t}{1+t} d t .
$$

i.e.

$$
\begin{aligned}
\frac{L^{m}[f](z)}{z} \prec q(z) & =\frac{1}{z} \int_{0}^{z} \frac{1+(2 \alpha-1) t}{1+t} d t \\
& =\frac{1}{z} \int_{0}^{z}\left[2 \alpha+1+\frac{2(1-\alpha)}{1+t}\right] d t \\
& =2 \alpha-1+2(1-\alpha) \frac{\ln (1+z)}{z}, z \in U .
\end{aligned}
$$

The function $q$ is convex and is the best dominant.

Since $q$ is a convex function and $p(z) \prec q(z), z \in U$, we have

$$
\operatorname{Re} p(z)>\operatorname{Re} q(1)=\delta=2 \alpha-1+2(1-\alpha) \ln 2 .
$$

Using (15), then (20) becomes

$$
\operatorname{Re} \frac{D^{m}[f](z)}{z}>\delta=2 \alpha-1+2(1-\alpha) \ln 2 .
$$

Using (7), we have

$$
\begin{gathered}
=\operatorname{Re} \frac{D^{m}[f](z)}{z} \\
=\operatorname{Re} \frac{z+\sum_{k=2}^{\infty} a_{k} z^{k}\left\{\left[\begin{array}{c}
m+k-1 \\
m
\end{array}\right](1-\lambda)+\lambda \frac{2^{m}}{(k+1)^{m}}\right\}}{z} \\
=\operatorname{Re}\left(1+\sum_{k=2}^{\infty} a_{k} z^{k-1}\left\{\left[\begin{array}{c}
m+k-1 \\
m
\end{array}\right](1-\lambda)+\lambda \frac{2^{m}}{(k+1)^{m}}\right\}\right)^{\prime} \\
=\operatorname{Re}\left(z+\sum_{k=2}^{\infty} \frac{a_{k}}{k} z^{k}\left\{\left[\begin{array}{c}
m+k-1 \\
m
\end{array}\right](1-\lambda)+\lambda \frac{2^{m}}{(k+1)^{m}}\right\}\right)^{\prime} \\
=\operatorname{Re}\left(z+\sum_{k=2}^{\infty} b_{k} z^{k}\left\{\left[\begin{array}{c}
m+k-1 \\
m
\end{array}\right](1-\lambda)+\lambda \frac{2^{m}}{(k+1)^{m}}\right\}\right)^{\prime} \\
=\operatorname{Re}\left[D^{m}[g](z)\right]^{\prime},
\end{gathered}
$$

where

$$
g(z)=z+\sum_{k=2}^{\infty} b_{k} z^{k}
$$


Using (22) in (21) we get

$$
\operatorname{Re} \frac{D^{m}[f](z)}{z}=\operatorname{Re}\left(D^{m}[g](z)\right)^{\prime}>\delta .
$$

Using Definition 4, we have $f \in M(m, \lambda, \delta)$, with $\delta$ given by (20). Since $f \in$ $M(m, \lambda, \alpha)$ was taken arbitrary and we have proved that $f \in M(m, \lambda, \delta)$, we conclude that $M(m, \lambda, \alpha) \subset M(m, \lambda, \delta)$.

From Theorem 2 we deduce the following corollary:

Corollary 1. If $f \in M(m, \lambda, \delta)$, then

$$
\operatorname{Re} \frac{D^{m}[f](z)}{z}>2 \alpha-1+2(1-\alpha) \ln 2=\delta .
$$

Proof. From the proof of Theorem 2, we can see that

$$
\begin{aligned}
\frac{L^{m}[f](z)}{z} \prec q(z) & =\frac{1}{z} \int_{0}^{z} \frac{1+(2 \alpha-1) t}{1+t} d t \\
& =2 \alpha-1+2(1-\alpha) \frac{\ln (1+z)}{z}, z \in U .
\end{aligned}
$$

Since $q$ is convex function, we have that

$$
\operatorname{Re} \frac{D^{m}[f](z)}{z}>\operatorname{Re} q(1)=2 \alpha-1+2(1-\alpha) \ln 2=\delta .
$$

Theorem 3. Let $h$ be a convex function in $U$ with $h(0)=1$.

If $f \in A, 0 \leq \lambda \leq 1, m \in \mathbb{N}$ and satisfies the differential subordination

$$
\left((1-\lambda) R^{m}[f](z)+\lambda L^{m}[f](z)\right)^{\prime} \prec h(z), z \in U
$$

then

and this result is sharp.

$$
\frac{(1-\lambda) R^{m}[f](z)+\lambda L^{m}[f](z)}{z} \prec q(z)=\frac{1}{z} \int_{0}^{z} h(t) d t
$$

Proof. We let

$$
p(z)=\frac{(1-\lambda) R^{m}[f](z)+\lambda L^{m}[f](z)}{z}, z \in U .
$$

Using (7) in (24), we obtain

$$
\begin{aligned}
p(z) & =\frac{z+\sum_{k=2}^{\infty} a_{k} z^{k}\left\{\left[\begin{array}{c}
m+k-1 \\
m
\end{array}\right](1-\lambda)+\lambda \frac{2^{m}}{(k+1)^{m}}\right\}}{z} \\
& =1+\sum_{k=2}^{\infty} a_{k} z^{k-1}\left\{\left[\begin{array}{c}
m+k-1 \\
m
\end{array}\right](1-\lambda)+\lambda \frac{2^{m}}{(k+1)^{m}}\right\} \\
& =1+p_{1} z+p_{2} z^{2}+\ldots, p \in \mathcal{H}[1,1] .
\end{aligned}
$$


From (24) we have

$$
(1-\lambda) R^{m}[f](z)+\lambda L^{m}[f](z)=z p(z), z \in U .
$$

Differentiating (25), we obtain

$$
\left((1-\lambda) R^{m}[f](z)+\lambda L^{m}[f](z)\right)^{\prime}=p(z)+z p^{\prime}(z), z \in U,
$$

then the differential subordination (23) becomes

$$
p(z)+z p^{\prime}(z) \prec h(z)=q(z)+z q^{\prime}(z), z \in U .
$$

By using Lemma $\mathrm{A}$, for $n=1, \gamma=1$, we have

$$
p(z) \prec q(z),
$$

i.e.

where

$$
\frac{(1-\lambda) R^{m}[f](z)+\lambda L^{m}[f](z)}{z} \prec q(z), z \in U,
$$

$$
q(z)=\frac{1}{z} \int_{0}^{z} h(t) d t
$$

and the function $q$ is convex and is the best dominant.

Theorem 4. Let $q$ be convex function in $U$, with $q(0)=1$, and let $\theta$ and $\varphi$ be analytic in a domain $D$ containing $q(U)$. Set

$$
Q(z)=z q^{\prime}(z) \varphi[q(z)] \text { and } h(z)=\theta[q(z)]+Q(z) .
$$

Let $m \in \mathbb{N}, f \in A, 0 \leq \lambda \leq 1$ and satisfies the differential subordination

$$
\frac{D^{m}[f](z)}{D^{m-1}[f](z)}+z\left(\frac{D^{m}[f](z)}{D^{m-1}[f](z)}\right)^{\prime} \prec q(z)+z q^{\prime}(z)
$$

then

$$
\frac{D^{m}[f](z)}{D^{m-1}[f](z)} \prec q(z), z \in U
$$

and $q$ is the best dominant. The operator $D^{m}[f]$ is defined in (6).

Proof. We let

$$
p(z)=\frac{D^{m}[f](z)}{D^{m-1}[f](z)}, z \in U
$$

Using (7) in (28) we obtain

$$
\begin{aligned}
p(z)= & \frac{z+\sum_{k=2}^{\infty} a_{k} z^{k}\left\{\left[\begin{array}{c}
m+k-1 \\
m
\end{array}\right](1-\lambda)+\lambda \frac{2^{m}}{(k+1)^{m}}\right\}}{z+\sum_{k=2}^{\infty} a_{k} z^{k}\left\{\left[\begin{array}{c}
m+k-1 \\
m
\end{array}\right](1-\lambda)+\lambda \frac{2^{m-1}}{(k+1)^{m-1}}\right\}} \\
= & 1+p_{1} z+p_{2} z^{2}+\ldots, p \in \mathcal{H}[1,1] .
\end{aligned}
$$


Differentiating (28) and after a short calculation, we obtain

$$
z p^{\prime}(z)=z\left(\frac{D^{m}[f](z)}{D^{m-1}[f](z)}\right)^{\prime}, z \in U
$$

and

$$
p(z)+z p^{\prime}(z)=\frac{D^{m}[f](z)}{D^{m-1}[f](z)}+z\left(\frac{D^{m}[f](z)}{D^{m-1}[f](z)}\right)^{\prime}, z \in U .
$$

Using (29), the subordination (27) becomes

$$
p(z)+z p^{\prime}(z) \prec q(z)+z q^{\prime}(z), z \in U .
$$

In order to prove the theorem, we shall use Lemma B.

For this, we show that the necessary conditions are satisfied.

Let the functions $\theta: \mathbb{C} \rightarrow \mathbb{C}$, and $\varphi: \mathbb{C} \rightarrow \mathbb{C}$, with

$$
\theta(w)=w, \varphi(w)=1 .
$$

We check the conditions from the hypothesis of Lemma B. Using (30), in the hypothesis of the theorem, we have

$$
Q(z)=z q^{\prime}(z) \varphi(q(z))=z q^{\prime}(z)
$$

and

$$
h(z)=\theta[q(z)]+Q(z)=q(z)+Q(z)=q(z)+z q^{\prime}(z), z \in U .
$$

Differentiating (31) and after short calculation, we obtain

$$
\frac{z Q^{\prime}(z)}{Q(z)}=1+\frac{z q^{\prime \prime}(z)}{q^{\prime}(z)}
$$

Since $q$ is convex function in $U$, we have

$$
\operatorname{Re} \frac{z Q^{\prime}(z)}{Q(z)}=\operatorname{Re}\left(1+\frac{z q^{\prime \prime}(z)}{q^{\prime}(z)}\right)>0, z \in U
$$

hence the function $Q$ is starlike.

Differentiating (32) and after short calculation, we obtain

$$
\frac{z h^{\prime}(z)}{Q(z)}=\frac{z q^{\prime}(z)+z Q^{\prime}(z)}{Q(z)}=\frac{z q^{\prime}(z)}{z q^{\prime}(z)}+\frac{z Q^{\prime}(z)}{Q(z)}=1+\frac{z Q^{\prime}(z)}{Q(z)} .
$$

Since $Q$ is starlikeness we have

$$
\operatorname{Re} \frac{z h^{\prime}(z)}{Q(z)}>0, z \in U .
$$

Using (30), we get $\theta[p(z)]=p(z), \varphi(p(z))=1$, then the subordination (27) becomes

$$
\theta[p(z)]+z p^{\prime}(z) \varphi[p(z)] \prec \theta[q(z)]+z q^{\prime}(z) \varphi[q(z)] .
$$

Using Lemma $\mathrm{C}$, we have

$$
p(z) \prec q(z),
$$


i.e.

$$
\frac{D^{m}[f](z)}{D^{m-1}[f](z)} \prec q(z), z \in U .
$$

Theorem 5. Let $q$ be a convex function $q(0)=1$, and let $\theta$ and $\varphi$ be analytic in a domain $D$ containing $q(U)$. Set

$$
Q(z)=z q^{\prime}(z) \varphi[q(z)] \text { and } h(z)=\theta[q(z)]+Q(z) .
$$

If $f \in A, \lambda \geq 0, m \in \mathbb{N}$, and satisfies the differential subordination

$$
\left(D^{m}[f](z)\right)^{\prime}+z\left(D^{m}[f](z)\right)^{\prime \prime} \prec h(z), z \in U,
$$

then

$$
\left(D^{m}[f](z)\right)^{\prime} \prec q(z), z \in U,
$$

and $q$ is the best dominant.

Proof. We let

$$
p(z)=\left(D^{m}[f](z)\right)^{\prime}, z \in U .
$$

Using (6) in (35), we get

$$
\begin{aligned}
p(z) & =\left(z+\sum_{k=2}^{\infty} a_{k} z^{k}\left\{\left[\begin{array}{c}
m+k-1 \\
m
\end{array}\right](1-\lambda)+\lambda \frac{2^{m}}{(k+1)^{m}}\right\}\right)^{\prime} \\
& =1+\sum_{k=2}^{\infty} k a_{k} z^{k-1}\left\{\left[\begin{array}{c}
m+k-1 \\
m
\end{array}\right](1-\lambda)+\lambda \frac{2^{m}}{(k+1)^{m}}\right\}, \\
& p(0)=1 \text { and } p \in \mathcal{H}[1,1] .
\end{aligned}
$$

Multiplying (35) by $z$, we obtain

$$
z p(z)=z\left(D^{m}[f](z)\right)^{\prime} .
$$

Differentiating (36), we get

$$
\left.\left.p(z)+z p^{\prime m}[f](z)\right)^{\prime m}[f](z)\right)^{\prime \prime}, z \in U .
$$

Using (37), the differential subordination (34) becomes

$$
p(z)+z p^{\prime}(z) \prec h(z), z \in U .
$$

In order to prove the theorem, we shall use Lemma B.

For this, we show that the necessary conditions are satisfied.

Let the function $\theta: \mathbb{C} \rightarrow \mathbb{C}$, and $\varphi: \mathbb{C} \rightarrow \mathbb{C}$, with

$$
\theta(w)=w \text { and } \varphi(w)=1 .
$$

We check the conditions from the hypothesis of Lemma B. Using (39) in the hypothesis of the theorem, we have

$$
Q(z)=z q^{\prime}(z) \varphi[q(z)]=z q^{\prime}(z)
$$


and

$$
h(z)=\theta[q(z)]+Q(z)=q(z)+Q(z)=q(z)+z q^{\prime}(z), z \in U .
$$

Differentiating (40), we have

$$
\frac{z Q^{\prime}(z)}{Q(z)}=1+\frac{z q^{\prime \prime}(z)}{q^{\prime}(z)}, z \in U
$$

Since $q$ is convex function,

$$
\operatorname{Re} \frac{z Q^{\prime}(z)}{Q(z)}>0, z \in U
$$

hence the function $Q$ is starlike.

Differentiating (41) and after short calculation, we obtain

$$
\frac{z h^{\prime}(z)}{Q(z)}=\frac{z q^{\prime}(z)+z Q^{\prime}(z)}{Q(z)}=\frac{z q^{\prime}(z)}{z q^{\prime}(z)}+\frac{z Q^{\prime}(z)}{Q(z)}=1+\frac{z Q^{\prime}(z)}{Q(z)} .
$$

Since $Q$ is starlike, we have

$$
\operatorname{Re} \frac{z h^{\prime}(z)}{Q(z)}=\operatorname{Re}\left(1+\frac{z Q^{\prime}(z)}{Q(z)}\right)>0, z \in U
$$

Using (39), we get

$$
\theta[p(z)]=p(z), \varphi[p(z)]=1,
$$

then the subordination (34) becomes

$$
\theta[p(z)]+z p^{\prime}(z) \varphi[p(z)] \prec \theta[q(z)]+z q^{\prime}(z) \varphi[q(z)] .
$$

Using Lemma B, we have i.e.

$$
p(z) \prec q(z),
$$

$$
\left(D^{m}[f](z)\right)^{\prime} \prec q(z), z \in U .
$$

Example 1. Let the convex function

$$
h(z)=\frac{1-z}{1+z}, h(0)=1,
$$

and

then

$$
f(z)=z+\frac{1}{2} z+\frac{1}{3} z^{3}, \lambda=\frac{1}{4}, m=1,
$$

$$
\frac{\left(1-\frac{1}{4}\right) R^{1}[f](z)+\frac{1}{4} L^{1}[f](z)}{z}=1+\frac{5}{6} z^{2}+\frac{19}{24} z^{3} .
$$

Using Theorem 3 we get:

If

$$
\frac{5}{3} z+\frac{19}{8} z^{2} \prec \frac{1-z}{1+z}
$$


then

$$
\begin{aligned}
1+\frac{5}{6} z^{2}+\frac{19}{24} z^{3} \prec q(z) & =\frac{1}{z} \int_{0}^{z} \frac{1-t}{1+t} d t=\frac{1}{z} \int_{0}^{z}\left(-1+\frac{2}{1+t}\right) d t \\
& =-1+\frac{2 \ln (1+z)}{z}
\end{aligned}
$$

and this result is sharp.

\section{REFERENCES}

[1] J. W. I. Alexander, "Functions which map the interior of the unit circle upon simple regions." Ann. Math. (2), vol. 17, pp. 12-22, 1915, zbMATH02616960, doi: 10.2307/2007212.

[2] D. J. Hallenbeck and S. Ruscheweyh, "Subordination by convex functions." Proc. Am. Math. Soc., vol. 52, pp. 191-195, 1975, doi: 10.2307/2040127.

[3] J. Krzyz, "A counterexample concerning univalent functions, folias soc." Scient. Lubliniensis, Mat. Fiz. Chem., vol. 2, pp. 57-58, 1962. [Online]. Available: https://ci.nii.ac.jp/naid/10010237867/en/

[4] T. H. MacGregor, "Functions whose derivative has a positive real part." Trans. Am. Math. Soc., vol. 104, pp. 532-537, 1962, https://doi.org/10.1090/S0002-9947-1962-0140674-7, zbMATH03172872, doi: 10.2307/1993803.

[5] S. S. Miller and P. T. Mocanu, "Second order differential inequalities in the complex plane." $J$. Math. Anal. Appl., vol. 65, pp. 289-305, 1978, https://doi.org/10.1016/0022-247X(78)90181-6, zbMATH03572508, doi: 10.1016/0022-247X(78)90181-6.

[6] S. S. Miller and P. T. Mocanu, "Differential subordinations and univalent functions." Mich. Math. J., vol. 28, pp. 157-171, 1981, zbMATH03685834, doi: 10.1307/mmj/1029002507.

[7] S. S. Miller and P. T. Mocanu, Differential subordinations: theory and applications. New York, NY: Marcel Dekker, 2000, vol. 225, zbMATH01392011.

[8] P. T. Mocanu, "Some starlikeness conditions for analytic functions." Rev. Roum. Math. Pures Appl., vol. 33, no. 1-2, pp. 116-124, 1988, zbMATH04063454.

[9] P. T. Mocanu and G. S. Sălăgean, Geometric Function Theory. Cluj-Napoca: Casa Cărţii de Ştiinţă, 1999.

[10] G. I. Oros, Using differential subordinations in the study of certain classes of univalent functions. Cluj-Napoca: Casa Cărţii de Ştiinţă, 2008.

[11] G. Oros, Convexity and starlikeness in geometric function theory. Budapest: Caretaken by the PAMM, Centre at the Bute, 2001.

[12] A. O. Páll-Szabó, "On a class of univalent functions defined by Sălăgean integro-differential operator." Miskolc Math. Notes, vol. 19, no. 2, pp. 1095-1106, 2018, zbMATH07065664, doi: 10.18514/MMN.2018.2457.

[13] A. O. Páll-Szabó, "Coefficient bounds and fekete-szego problem for new classes of analytic functions defined by sălăgean integro-differential operator," Acta Univ. Apulensis, Math. Inform., vol. 57, pp. 147-158, 2019.

[14] S. Ruscheweyh, "New criteria for univalent functions." Proc. Am. Math. Soc., vol. 49, pp. 109-115, 1975, https://doi.org/10.1090/S0002-9939-1975-0367176-1, zbMATH03473988, doi: $10.2307 / 2039801$.

[15] H. M. Srivastava, S. Hussain, A. Raziq, and M. Raza, "The fekete-szegö functional for a subclass of analytic functions associated with quasi-subordination," Carpathian Journal of Mathematics, vol. 34, no. 1, pp. 103-113, 2018. [Online]. Available: http://www.jstor.org/stable/90021608 
[16] H. Srivastava, A. Prajapati, and P. Gochhayat, "Third-order differential subordination and differential superordination results for analytic functions involving the srivastava-attiya operator," Applied Mathematics and Information Sciences, vol. 12, pp. 469-481, 05 2018, doi: 10.18576/amis/120301.

[17] H. M. Srivastava, A. Motamednezhad, and E. A. Adegani, "Faber polynomial coefficient estimates for bi-univalent functions defined by using differential subordination and a certain fractional derivative operator," Mathematics, vol. 8, no. 2, p. 172, 2020.

[18] H. M. Srivastava, D. Răducanu, and P. Zaprawa, "A certain subclass of analytic functions defined by means of differential subordination," Filomat, vol. 30, no. 14, pp. 3743-3757, 2016.

[19] Q.-H. Xu, H.-G. Xiao, and H. M. Srivastava, "Some applications of differential subordination and the dziok-srivastava convolution operator," Applied Mathematics and Computation, vol. 230, pp. 496-508, 2014.

\section{Author's address}

\section{Georgia Irina Oros}

University of Oradea, Faculty of Sciences, Department of Mathematics and Computer Sciences, Str. Universitatii, No.1, 410087 Oradea, Romania

E-mail address: georgia_oros_ro@yahoo.co.uk, ORCID: 0000-0003-2902-4455 